\title{
ON THE ROLE OF MATHEMATICAL ABSTRACTIONS FOR SCIENTIFIC COMPUTING*
}

\author{
Krister Åhlander, Magne Haveraaen, Hans Z. Munthe-Kaas \\ University of Bergen \\ Bergen, Norway
}

\begin{abstract}
A distinguished feature of scientific computing is the necessity to design software abstractions for approximations. The approximations are themselves abstractions of mathematical models, and the mathematical models are also abstractions of the real world.

In this paper, the relation between different mathematical abstraction levels and scientific computing software is discussed, in particular with respect to the simulation of partial differential equations. By applying software engineering practices already when the mathematical model is considered, a coordinate-free formulation is readily motivated.

For partial differential equations, the continuos layer is identified as separate from the the coordinate-free layer. By mapping these layers into different software modules, numerical discretization carried out in the continuous layer is cleanly separated from the coordinate-free layer. This separation of concerns increases modularity because reusability is promoted.

It is therefore concluded that the continuous and coordinate-free abstraction layers provide a solid foundation for software that simulates partial differential equations.
\end{abstract}

Keywords: Abstraction, coordinate-free numerics, object-oriented modeling, PDE simulation.

\section{INTRODUCTION}

Applications in the area of scientific computing span a wide range of problem domains, and each domain exhibits its own challenges to be addressed. However, one distinctive feature of scientific computing applications is the need to deal with approximations of continuous structures.

\footnotetext{
${ }^{*}$ Research funded via a grant from the Norwegian research council.
} 
The obvious example is that the infinite number of reals on any interval must be approximated on a computer, normally by floating point numbers of appropriate precision. But even this basic example shows that scientific computing is a science where nothing is certain, since it is also possible to use, say, integers to represent real values, as is often done in image processing. Another example of a continuous approximation is the abstraction of an angle. In order to become a useful component, this abstraction must recognize that different real numbers, say $\pi$ and $3 \pi$, may represent the same angle, and also that different units such as radians or degrees may be used. These facts are of a different nature than the fact that the same real number may be approximated by different discrete representations. The understanding of abstractions and their approximations is a key aspect for scientific computing, in order to obtain modular software architectures.

In this paper, we promote the philosophy that the design of applications for scientific computing should be based firstly upon continuous abstractions and secondly on approximations. This is nicely illustrated by the angle abstraction having some continuous features, independent of a particular choice of real number approximation. An angle abstraction should also be general with respect to the choice of units. The same observation applies to physical quantities. A velocity vector, for instance, should be independent of any particular unit or any particular coordinate system. Consequently, we identify two different mathematical abstraction levels, a continuous abstraction level and a coordinate-free abstraction level.

The aim of this paper is to discuss the role mathematical abstractions have concerning the modularity of the software architecture. We focus on the simulation of partial differential equations (PDEs). The problem domain of PDE solvers is demanding, since it includes the need to evaluate approximate solutions of mathematical models, a request for high performance, and the necessity to utilize physically motivated simplifications where possible. In the Sophus project $[9,11]$ we find that a design which is based upon continuous structures is more modular than a design based upon discrete approximations of the continuous structures. A software architecture based on coordinate-free abstractions - coordinatefree numerics [15] - has additional flexibility. Here, PDEs can be written independent of the number of space dimensions and the choice of coordinate system, two properties that often change in applications.

There are many related initiatives in the scientific computing community that address software abstractions for PDE solvers, for instance $[1,5,6,20]$. The emphasis on continuous abstractions is also noted in [16] regarding the modeling of computational geometries. For 
geometric integration [12] of ordinary differential equations, Diffman provides a coordinate-free software package [7]. Coordinate-free numerical optimization is also emphasized in [8]. To some extent, coordinate-free differentiation of PDEs is also addressed in Overture [4] concerning composite curvilinear grids.

The remainder of this paper is organized as follows. In Section 2 we discuss some mathematical abstractions and introduce the notion of different mathematical abstraction levels, giving examples from the area of PDEs. In Section 3 we note a few implications for software architecture. Our concluding section points out that coordinate-free mathematical concepts are essential for the modularity of PDE simulating software.

\section{MATHEMATICAL ABSTRACTIONS}

In software engineering, a number of object-oriented methodologies have been advocated in order to obtain software systems with high reliability, robustness, modularity, understandability, and so forth. See for example [3, 13, 17]. Some of the most important principles used in object-oriented modeling are information hiding, abstraction, polymorphism, and aggregation. Moreover, as a general guide-line, the software is to be based on concepts in the real world that we want to model.

In the context of PDE solvers, these principles imply that the software abstractions must come from the mathematical domain of PDEs. This is recognized by all relevant software projects, including those previously mentioned in the introduction. However, we believe that the modularity of the final application is highly dependent on which mathematical abstractions one chooses as input for the design. In order to choose appropriate mathematical abstractions, we think that software engineering practices must be applied already when modeling the mathematical domain. In this section we illustrate this idea and we motivate coordinatefree numerics by pure continuous considerations, see Section 2.4.

As a first step we divide our problem domain of PDEs into different layers and subsystems. These partitions should ideally correspond to software modules, aiming for a good modularity. We identify the following levels:

1 Manifold abstractions

2 Functions on manifolds and partial derivatives

3 Coordinate-free tensor abstractions

4 Equation abstractions 
Below, we discuss these levels further. Our presentation will recapitulate some basic mathematics for PDEs, mostly from a coordinate-free point of view. For a more thourough introduction, we recommend [19].

\subsection{MANIFOLD ABSTRACTIONS}

The manifold is a central concept for PDEs. The manifold is the space where the entities of the PDE are defined and where the solution of the PDE evolves. Examples of manifolds are three-dimensional space, four-dimensional space-time, phase-space, or the sphere. We want to study the common continuous properties of manifolds, because, as Shutz notes $[19$, p. 23]: "All these spaces have different geometrical properties, but they all share something in common, something which has to do with their being continuous spaces rather than, say, lattices of discrete points."

Mathematically, a manifold $M$ with dimension $n$ is defined as a set of points where each point of $M$ has an open neighborhood which has a continuous 1-1 map onto an open set of $\Re^{n}$. Thus, every manifold is locally 'like' $\Re^{n}$. On a very "primitive" manifold, the notion of distance is not needed, but we will assume that we deal with Riemannian manifolds with a metric.

\subsection{MANIFOLD FUNCTIONS AND PARTIAL DERIVATIVES}

A PDE is a relation between various tensor fields and their derivatives. In this section, we first discuss the auxiliary concept of a "manifold function". We then discuss partial derivatives.

- A manifold function Manifold $\mathrm{F} c n<\mathrm{T}>$ is parametrized over a type $T$. It is defined as having a value of type $T$ in every point of a particular manifold. It can be thought of as a continuous array with the same dimension as the manifold. The type $\mathrm{T}$ must have all the usual arithmetic operations, addition, multiplication etc. These operations may the be "lifted" pointwise from type $\mathrm{T}$ to type Manifold $F(n<T\rangle$. This corresponds to a significant raise of abstraction level, allowing us to simultaneously manipulate all values in a Manifold $\mathrm{F} C n<\mathrm{T}>$ object.

- In order to treat partial derivatives, we introduce PD scalar field, a scalar field which only has partial derivatives. It can be thought of as Manifold Fcn<Scalar> with additional operations for partial differentiation. Thus, the first category of PD scalar field operations is the same as for manifold functions, obtained by lifting 


\begin{tabular}{|l|l|l|l|}
\hline TensorField & \multicolumn{2}{|l|}{ Tensor } \\
\hline TensorField op+(TensorField) & p:Point & & Tensor op+(Tensor) \\
TensorField apply(TensorField) & & \\
TensorField Lie(TensorField) \\
TensorField div()
\end{tabular}

Figure 1 Relations between different abstractions for PDEs. The PD scalar field consists of several scalars, one for each point in the manifold. A tensor has several scalar components. For a tensor field $t f$, we require $t f[p][c]=t f[c][p]$, and a tensor field can be seen either as having a tensor in each point or a scalar field for every component.

arithmetic operations on scalars. The second category, the differentiation operations, study how the scalar field changes between different points. PD scalar field is illustrated in the lower part of Figure 1 using UML [18]. We have exemplified arithmetic operations with the + operator, and we denote with ddx partial derivative operation, $\partial / \partial x^{i}$.

\subsection{COORDINATE-FREE ABSTRACTIONS}

The principle that the underlying physical laws shall be independent on the choice of coordinate system is fundamental for our understanding of the world. Therefore, it is beneficial to use coordinate-free formulations of the laws. Such formulations are based on tensors and derivatives of tensors.

Briefly, a tensor of order $n$ in a point $p$ of a manifold with dimension $d$ can be represented with $d^{n}$ components. The basic arithmetic operations must hold for its components. As for manifold functions, arithmetic operations may be component-wise lifted to tensors. Moreover, a tensor may be applied to another tensor, yielding a third tensor. Tensors can 
therefore be regarded as multi-linear mappings between tensor spaces. See equation (1.2) below for an example. Familiar examples of tensors are vectors, which have order 1 , and scalars, which have order 0 .

A tensor field is like a Manifold Fcn<Tensor $>$ with additional operations for coordinate-free derivation. The operations of interest in the tensor field interface come from the same categories as for the scalar field: the lifting of operations from the tensor abstraction, and derivatives. In the top of Figure 1, we have listed some coordinate-free derivatives in the Tensor field interface. A Lie derivative has as a parameter a tensor field of order 1 , a vector field. We have also listed div $=\nabla \cdot$, the divergence. In order to compute the coordinate-free derivatives, the tensor field uses the metric tensor which holds information about the coordinate system.

Regarding the lifting of arithmetic operations, we note in Figure 1 that we can lift for instance the + operator either from the tensor or from the scalar field. These results must be identical.

\subsection{EQUATION PACKAGES}

Above, we have concentrated on mathematical definitions of basic mathematical concepts. Here, we compare the coordinate-free approach with a component dependent approach.

As a motivating example, we use the wave equation for an elastic medium. A typical industrial application of the wave equation is to simulate seismic waves in oil reservoirs [2]. It is a standard equation from mathematical physics, and may be found in any text book on the subject, for instance [14]. In its most compact version, the coordinatefree form, it may be stated as:

$$
\begin{aligned}
\rho \frac{\partial^{2} u}{\partial t^{2}} & =\nabla \cdot \sigma+f(t) \\
\sigma & =\Lambda(e) \\
e & =\mathcal{L}_{u} g .
\end{aligned}
$$

Here, $\rho$ is a scalar field, $u$ is the displacement vector to be simulated, $f$ is a time-dependent forcing function, $\sigma, e, \Lambda$ and $g$ are tensors of order two $(\sigma, e$ and $g)$ and four $(\Lambda)$. The equations also involve derivation with respect to time, $\frac{\partial}{\partial t}$, the divergence $\nabla$. and the Lie derivative $\mathcal{L}_{u}$.

In order to interpret the equations, we may assume Cartesian coordinates, and the equations may then be reformulated in component form as:

$$
\rho \frac{\partial^{2} u_{i}}{\partial t^{2}}=\sum_{k} \frac{\partial \sigma_{i k}}{\partial x_{k}}+f_{i}(t)
$$




$$
\begin{aligned}
\sigma_{i j} & =\sum_{k l} \Lambda_{i j k l} e_{k l} \\
e_{i j} & =\frac{1}{2}\left(\frac{\partial u_{i}}{\partial x_{j}}+\frac{\partial u_{j}}{\partial x_{i}}\right) .
\end{aligned}
$$

Here, the component indices $i$ and $j$ and the summation indices $k$ and $l$ vary over the number of space dimensions.

Sometimes, the model we simulate is axi-symmetric. When simulating oil reservoirs, this is a typical assumption in the vicinity of a bore hole. The problem may then be simplified in cylindrical coordinates by observing that $u(r, \theta, z)$ is independent of $\theta$. The change of coordinate system has implications for the metric tensor and thus the derivatives involved. The coordinate-free formulation remains intact, but the component form must be reformulated. Introducing auxiliary functions $d_{i}$ and $L_{i j}$ for the derivatives in cylindrical coordinates, we write

$$
\begin{aligned}
\rho \frac{\partial^{2} u_{i}}{\partial t^{2}} & =d_{i}+f_{i} \\
\sigma_{i j} & =\sum_{k l} \Lambda_{i j k l} e_{k l} \\
e_{i j} & =L_{i j}
\end{aligned}
$$

To keep the presentation simple, we do not present explicit formulas for the derivatives. The point is that the formulas change. One can derive, for example, that $L_{\theta \theta}$ in this case is given by $u_{r} / r$, and not by $\partial u_{\theta} / \partial \theta$, as equation (2.3) suggests.

The fact that the coordinate-free formulation remains intact is the key motivation to the coordinate-free approach. We believe the invariance of (1) to be significant. We illustrate the situation in Figures 2 and 3. These UML diagrams depict two tentative UML models of the wave equation. We believe that already in this early stage of the design process, the two models have significant differences with respect to modularity.

Figure 2 illustrates a coordinate-free mathematical model. We model the wave equation (1) as an aggregate of its three equations. Each individual equation is expressed using tensors. These tensors are associated with the metric of the manifold, needed for computing differentiation expressions.

Figure 3 illustrates the component equations (2) and (3). The main difference is that a coordinate dependent interface is used to express the equations, and the equations are explicitly formulated using partial derivatives. In order to represent both Cartesian coordinates and 


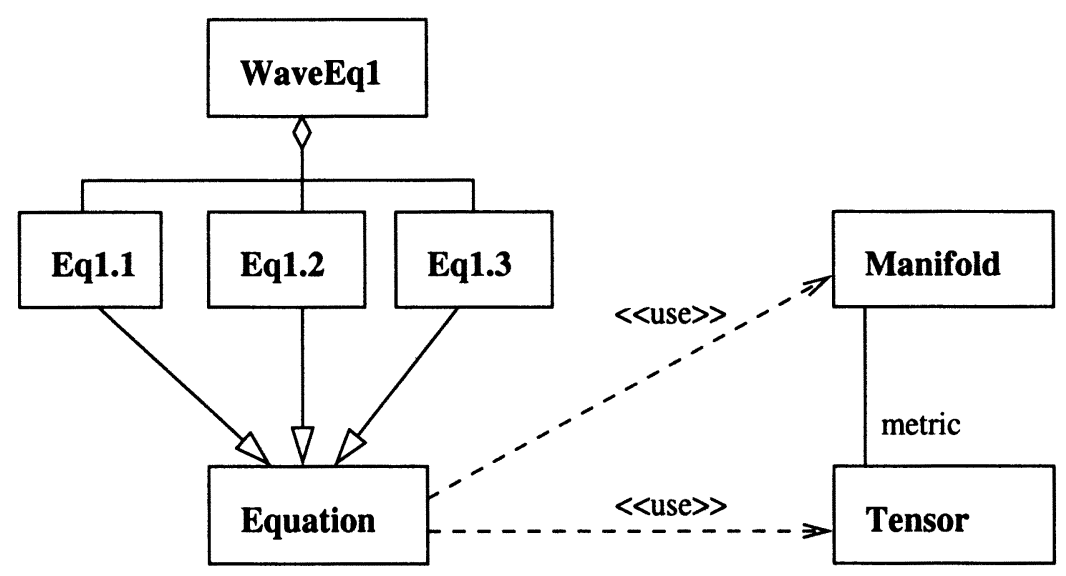

Figure 2 A coordinate free mathematical model is based upon equations that use a coordinate-free interface to tensor fields. A change of coordinates affect the tensor metric, but the equations remain untouched.

cylindrical coordinates, we are now forced to have different versions for equations in different coordinate systems. Even if the equation $\sigma_{i j}=\sum_{k l} \Lambda_{i j k l} e_{k l}$ is invariant under a change of coordinate system, we mean that the dependence of equations on the coordinate system reflects a poor model, in the sense that it is sensitive to changes. The coordinate-free diagram, on the other hand, represents a more robust model. This model is better suited as input for software design.

\section{IMPLICATIONS FOR THE SOFTWARE ARCHITECTURE}

In earlier sections, we have discussed modeling of the PDE domain, from a continuous perspective. We have shown the importance of software engineering concepts already at this early stage of the design process. In this section, we discuss the transition from the continuous model to a discrete model, necessary in order to simulate PDEs on a computer. The final software design is of course dependent on the choice of discretization techniques. We believe, however, that if the design is based on the continuous and coordinate-free abstractions presented earlier, a software architecture for PDE solvers can be obtained which is robust with respect to different choices of coordinates and discretization techniques. 


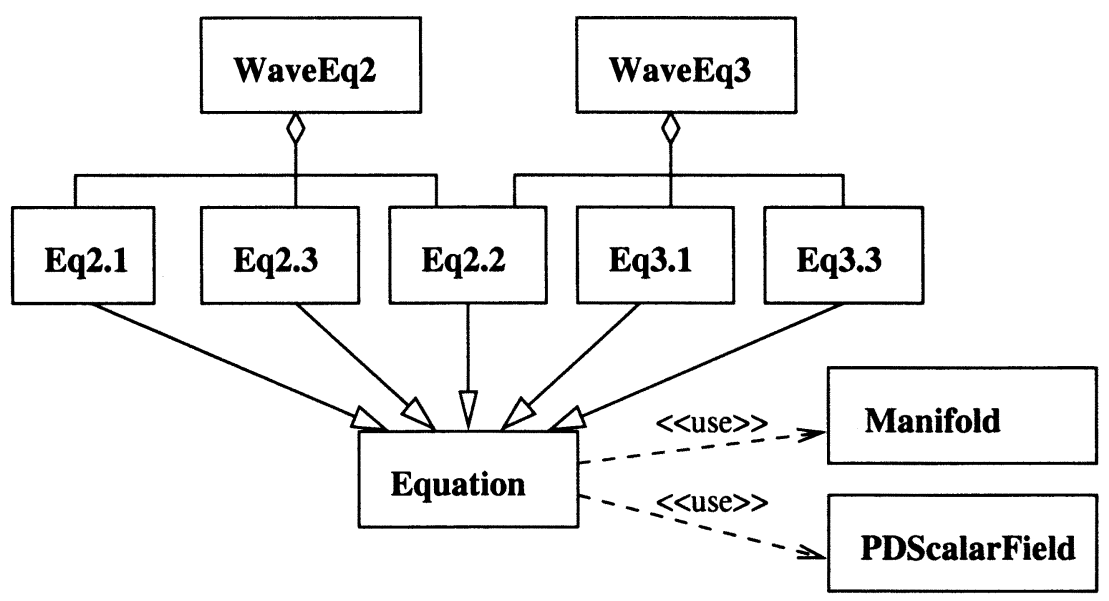

Figure 3 A mathematical model based on partial derivatives makes the equations coordinate dependent. The metric of the manifold is in this case intermingled with the equation formulation. Note that (2.2) is the same equation as (3.2), which is why WaveEq3 reuses Eq2.2.

Table 1 Different continuous abstractions lead to different discrete abstractions.

\begin{tabular}{ll}
\hline Continuous & Discrete \\
\hline$u=u(\mathbf{x})$ & $u_{i j}=u\left(x_{i j}\right)$ \\
$u=\sum_{l=1}^{\infty} c_{l} \Psi_{l}(\mathbf{x})$ & $u=\sum_{l=1}^{N} c_{l} \Psi_{l}(\mathbf{x})$
\end{tabular}

\subsection{DISCRETIZATION}

When discretizing, it is important that the discrete model represents the continuous model. Several choices has to be made, and we exemplify here that different continuous models yield different discrete models.

Consider a scalar field $u=u(\mathbf{x}), \mathbf{x} \in \mathcal{M} \subset \Re^{2}$. Of course, we can discretize the manifold, thus obtaining a discrete scalar field $u=u\left(x_{i j}\right)$. Another continuous model of a scalar field is to treat it as an infinite linear combination of basis functions: $u=\sum_{l=1}^{\infty} c_{l} \Psi_{l}$. By terminating the infinite series, see Table 1, we discretize using a completely other mechanisms. The two different mechanisms may be observed to correspond to rounding (discretizing the underlying manifold) and truncation (terminating an infinite series).

Most PDE solvers discretize scalar fields and tensor fields by "lifting" a discretization of the manifold. Many projects, including Sophus [10], 
provides both serial and parallel versions of the manifold. In this respect, the manifold is proven to be a reusable and flexible software module. However, it is not easy to mix manifold modules from different projects.

Besides discretizing the manifold, discretization of derivatives must also be addressed. According to our results from the previous section, PD scalar field is the proper module for computing derivatives. With a proper interface, we may easily provide different implementations. For example, Sophus provides both standard finite differences and finite differences for a staggered grid, see Figure 4.

\subsection{TENSOR FIELD ABSTRACTIONS}

Compared with PD scalar fields, tensor fields are on a higher level of abstraction. Developing a good tensor module is a challenging task. Still, in one respect the tensor field is "easier" to implement, since it can be developed without explicit reference to the discretization. Achieving this goal requires some care, as discussed in this section.

Considering Figure 1 on page 149 again, we note that there are two seemingly equivalent ways to represent tensors, either as an aggregate of scalar fields or as an aggregate of tensors. As a concrete example, we consider a vector field on a two-dimensional manifold:

$$
\mathbf{u}(x, y)=\left(u_{1}(x, y), u_{2}(x, y)\right) .
$$

It can be regarded as an "array" with three indices. The first is discrete and picks out the component, and the other two "indices" are the continuous coordinates. A discrete representation, based on Cartesian coordinates, would also give a three-dimensional array structure: $u_{i j l}=u_{l}\left(x_{i}, y_{j}\right)$. It seems as if we have two equivalent ways to construct tensor field data:

$$
\text { ManifoldFcn<Tensor }<\text { Scalar }>>
$$

and

$$
\text { Tensor<ManifoldFcn<Scalar }>\text {. }
$$

In other words, we can either construct data for a Tensor Field as a ManifoldFcn over a Tensor or as a Tensor over a ManifoldFcn.

To spot the difference, we must take derivatives into account. If construct (4) would be used, all differentiation on the tensor field would have to be developed from scratch. This is related to the fact that two tensors in different points belong to different tensor spaces. The consequence is that if we change the underlying discretization of partial derivatives, all code concerning derivatives is changed in the tensor module. 


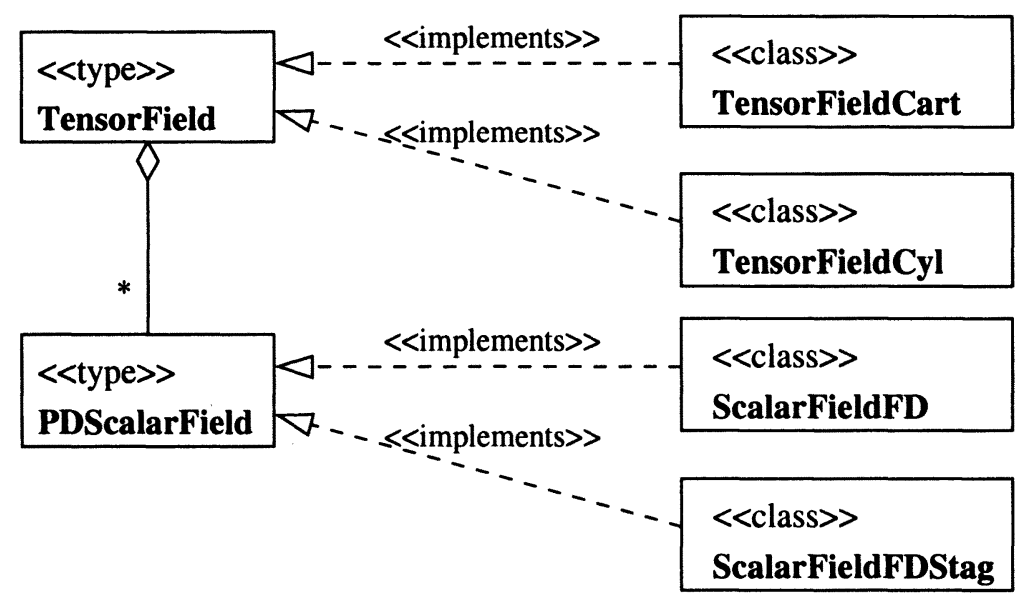

Figure 4 Different numerical discretizations may be supported with different scalar field implementations. Similarly, efficient tensor implementations for common coordinate systems may be provided.

The second construct is more robust.We introduce PD scalar field in place of ManifoldFcn. A Tensor<PDScalarField $>$ need to know the metric of the manifold in order to compute the coordinate-free derivatives, but the numerical discretizations are hidden from the tensor field abstraction. Division of responsibilities is crucial for modular software.

In practical computations, the computations can often be simplified if the metric is given. It may therefore be interesting to implement special purpose tensors for commonly used coordinate systems, see Figure 4. The discrete differentiation is taking place only within the discrete PD scalar fields. The tensor abstraction involves components, which are inherent in the continuous domain.

\section{CONCLUDING REMARKS}

The role of mathematical abstractions is discussed, and we argue that the correct treatment of these are important in order to design modular software architectures for scientific computing.

Particularly, we investigate the role of mathematical abstractions in the context of simulating partial differential equations, PDEs. By emphasizing software engineering practices already when analyzing the mathematical domain, we identify several levels of mathematical abstractions and subdomains. Obtaining a robust mathematics model is the first step towards a sound software architecture. 
These ideas have been used for designing Sophus, a PDE solver library built upon various mathematical abstractions. Particularly, we point out the separation of concerns between the tensor field module and the partial derivatives module. The former belongs to the coordinate-free layer and is the module where the metric of the manifold is used. The latter belongs to the continuous layer and is the module where numerical discretization of derivatives is performed. This separation of concerns is the key for achieving high modularity.

We find mathematical abstractions useful for the design of flexible PDE solvers. It would also be of interest to investigate the role of mathematical abstractions in other domains of scientific computing, for example visualization, optimization, or statistics.

\section{References}

[1] K. Åhlander. An Object-Oriented Framework for PDE Solvers. $\mathrm{PhD}$ thesis, Uppsala University, Dept. of Scientific Computing, Uppsala, Sweden, 1999.

[2] K. Aki and P. G. Richards. Quantitative Seismology Theory and Methods, volume 1. W.H.Freeman and Company, 1980.

[3] G. Booch. Object-Oriented Analysis and Design with Applications. Benjamin/Cummings, Reading, Massachusetts, 2nd edition, 1994.

[4] D. Brown, W. Henshaw, and D. Quinlan. Overture: An objectoriented framework for solving partial differential equations on overlapping grids. In S. L. L. M. E. Henderson and C. R. Anderson, editors, Object-oriented Methods for Interoperable Scientific and Engineering Computing, ch. 26, Philadelphia, 1999. SIAM.

[5] A. M. Bruaset and H. P. Langtangen. A comprehensive set of tools for solving partial differential equations; Diffpack. In M. Dæhlen and A. Tveito, editors, Numerical Methods and Software Tools in Industrial Mathematics, pages 61-90. Birkhäuser, Boston, 1997.

[6] J. C. Cummings et al. Rapid application development and enhanced code interoperability using the POOMA framework. In S. L. L. M. E. Henderson and C. R. Anderson, editors, Objectoriented Methods for Interoperable Scientific and Engineering Computing, ch. 29, Philadelphia, 1999. SIAM.

[7] K. Eng $\varnothing$, A. Marthinsen, and H. Z. Munthe-Kaas. DiffMan - an object oriented MATLAB toolbox for solving differential equations on manifolds. Technical Report No. 164, Dept Comp. Sc., University of Bergen, 1999. See also http://www.ii.uib.no/diffman. 
[8] M. S. Gockenbach, M. J. Petro, and W. W. Symes. C++ classes for linking optimization with complex simulations. ACM Transactions on Mathematical Software, 25(2):191-212, June 1999.

[9] P. Grant, M. Haveraaen, and M. Webster. Coordinate free programming of computational fluid dynamics problems. Scientific Programming, 2000. Accepted for publication.

[10] M. Haveraaen. Abstractions for programming parallel machines. Scientific Programming, 2000. Accepted for publication.

[11] M. Haveraaen, H. A. Friis, and T. A. Johansen. Formal software engineering for computational modeling. Nordic Journal of Computing, 6(3):241-270, 1999.

[12] A. Iserles et al. Lie-group methods. Acta Numerica, 9:215-365, 2000.

[13] I. Jacobsson, M. Christersson, P. Jonsson, and G. Övergaard. Object-Oriented Software Engineering. Addison-Wesley, Wokingham, England, 1992.

[14] J. E. Marsden and T. J. R. Hughes. Mathematical Foundations of Elasticity. Prentice-Hall, 1983.

[15] H. Munthe-Kaas and M. Haveraaen. Coordinate free numerics - closing the gap between 'pure' and 'applied' mathematics? Zeitschrift fuer Angewandte Mathematik und Mechanik, 76, supplement 1:487-488, 1996.

[16] K. Ottmar and M. Shephard. Embedding reliable numerical analysis capabilities into an enterprise wide information system. Submitted to Engineering with Computers, 2000.

[17] J. Rumbaugh et al. Object-Oriented Modeling and Design. PrenticeHall, Englewood Cliffs, New Jersey, 1991.

[18] J. Rumbaugh, I. Jacobson, and G. Booch. The Unified Modeling Language Reference Manual. Addison Wesley Longman, Reading, Massachusetts, 1999.

[19] B. Schutz. Geometrical Methods of Mathematical Physics. Cambridge University Press, 1980.

[20] M. Thuné et al. Object-oriented construction of parallel PDE solvers. In E. Arge, A. M. Bruaset, and H. P. Langtangen, editors, Modern Software Tools for Scientific Computing, pages 203-226. Birkhäuser, 1997. 


\section{DISCUSSION}

\section{Speaker: Krister Åhlander}

Robert van de Geijn : One interpretation of your talk is that a code implementing some component of a scientific computation should be more than an implementation of the solution method. It should be designed, structured and implemented to encode domain knowledge.

Krister Åhlander : I agree in this interpretation. Perhaps an additional point of the talk is that already the choice of mathematical abstractions influence the structure and design of the software domain.

Scott Kohn : At what point do concrete implementation details enter the abstraction? This is a very difficult question in scientific computing. I'm not sure that anyone has the answer.

Krister Åhlander : At the application level, we avoid implementation details. Concrete implementation details are coded in lower level modules, tensors, scalar fields, meshes. These are chosen at link time. As a remark, we think that compile time polymorphism is important in order to achieve high performance.

Reagan Moore : How does one guarantee numerical accuracy when the manifold, coordinate system, and physical parameters required for solving a differential equation must be simultaneously discretized.

Krister Åhlander : The purpose of Sophus is to separate different discretizations in different modules. We do not guarantee that a chosen module configuration is appropriate from a numerical point of view, but we provide the possibility to freely choose among different configurations. John R. Rice : A comment on previous question and answer: The difficulties here are illustrated by a simulation of the behavior of a small fluid droplet. The droplet surface is changing and its smoothness has a strong effect on behavior because of surface tension effects. To obtain physically correct results one must start with a mathematical representation of smooth surfaces that change smoothly. People who have started with a discretization of space into a grid or mesh have failed because their constructions introduced small deformations in the surface which are quickly magnified by surface tension. The mathematical representation of smooth manifolds which can vary easily is not a simple or well known technology, but it can be done. A multiple droplet environment where surfaces merge or divide is even more challenging both mathematically and in the physics. Nevertheless, it is essential to start with a high level abstraction of the drop surfaces. 